\title{
Effects of slip on free convection flow of Casson fluid over an oscillating vertical plate
}

\author{
Muhammad A Imran ${ }^{1 *}$, Shakila Sarwar ${ }^{2}$ and Muhammad Imran ${ }^{3}$
}

"Correspondence: imranasjad11@yahoo.com 'Department of Mathematics, University of Management and Technology, Lahore, Pakistan Full list of author information is available at the end of the article

\section{Introduction}

There has been great deal of interest in understanding the behavior of non-Newtonian fluids [1, 2]. Examples of such rheological complex fluids are blood plasma, chocolate, mustard mayonnaise, tooth paste, shampoo, food stuffs, mud, polymer melts, clay coatings, oils and greases, paints etc. These kinds of fluids offer special challenges to the engineers, modelers, mathematicians, and physicists. The study of non-Newtonian fluids is very important in view of its applications in various branches of engineering and technology, therefore the flow analysis of these fluids is very important in theory and practice. From a theoretical point of view, flows of this type are fundamental in fluid mechanics. Practically speaking, these flows have applications in many manufacturing processes in industry. Due to the great diversity in the physical structures of non-Newtonian fluids, it is not possible to establish a single constitutive equation. Thus, many non-Newtonian fluid models have been proposed of which most are empirical or semi-empirical. The equations of motion for non-Newtonian fluids are much more complicated and are of higher order than the Navier-Stokes equations. The solutions of most of the problems in the real world are usually even numerical, on computers. However, analytical solutions, even if they may not be accurate, can provide some penetrating insight into the physics of a problem which manages a maze of numbers crunched on a computer. For that reason, researchers still look for analytical solutions of the known and unknown problems, particularly the for- 
mer, as they can act as a bench mark for the latter. Various analytical and numerical approaches/methods by a number of people with and without slip condition [3-26] have been done.

Different models are suggested to express the constitutive equations of non-Newtonian fluids. Amongst these fluid models, there is one known as Casson fluid which was originally introduced by Casson [27]. We can define a Casson fluid as a shear thinning liquid which is assumed to have infinite viscosity at zero rate of shear, and a yield stress below which no flow occurs and a zero viscosity at an infinite rate of shear. The non-linear Casson's constitutive equation has been found to describe accurately the flow curves of suspensions of pigments in lithographic varnishes used for preparation of printing inks. In particular, the Casson fluid model describes the flow characteristics of blood more accurately at low shear rates and when it flows through small blood vessels [28]. Some famous examples of the Casson fluid include jelly, tomato sauce, honey, soup, and concentrated fruit juices etc. Many researchers [29-38] studied the Casson fluid under different boundary conditions. Some find the solutions by using either approximate methods or numerical schemes and some find its exact analytical solutions. The solutions when the Casson fluids are in free convection flow with constant wall temperature are also determined. On the other hand the flow of the Casson fluid in the presence of heat transfer is also an important research area. Motivated by Khalid et al. [39] we focused on the unsteady flow of a Casson fluid past an oscillating vertical plate with constant wall temperature under the non-slip conditions. In the present paper, we extended the work of Khalid et al. by applying the slip condition at the boundary. Exact solutions are obtained by applying the Laplace transform technique and it is found that the results in the absence of slip are fully agreed with that of [39].

\section{Statement of the problem}

Let us consider the heat transfer effect on unsteady boundary layer flow in a Casson fluid past an infinite oscillating vertical plate fixed at $y=0$, the flow being confined to $y>0$, where $y$ is the coordinate axis normal to the plate. Initially, for time $t=0$, both plate and fluid are under stationary conditions with the temperature $T_{\infty}$. At time $t=0^{+}$, the plate started an oscillatory motion in its plane and slip is considered at the boundary.

At the same time, the heat transfer from the plate to the fluid is proportional to the local surface temperature $T$. We assume that the rheological equation for an isotropic and incompressible Casson fluid, reported by Casson [27], is

$$
\tau=\tau_{o}+\mu \dot{\sigma},
$$

or

$$
\begin{aligned}
\tau_{i j} & =\left\{2\left(\mu_{B}+\frac{p_{y}}{\sqrt{2 \pi}}\right) e_{i j}, \pi>\pi_{c}\right\} \\
& =\left\{2\left(\mu_{B}+\frac{p_{y}}{\sqrt{2 \pi_{c}}}\right) e_{i j}, \pi_{c}<\pi\right\},
\end{aligned}
$$

where $\tau$ is the shear stress, $\tau_{o}$ is the Casson yield stress, $\mu$ is the dynamic viscosity, $\dot{\sigma}$ is the shear rate, $\pi=e_{i j} e_{i j}$, and $e_{i j}$ is the $(i, j)$ th component of the deformation rate, $\pi$ is the product of the component of deformation rate with itself, $\pi_{c}$ is a critical value of this 
product based on the non-Newtonian model, $\mu_{B}$ the is plastic dynamic viscosity of the non-Newtonian fluid and $p_{y}$ is the yield stress of the fluid. The velocity as well as the temperature are functions of $y, t$ only.

Under the Boussinesq approximation along with the assumption that the pressure is uniform across the boundary layer, we get the following set of partial differential equations [39]:

$$
\begin{aligned}
& \frac{\partial u(y, t)}{\partial t}=v\left(1+\frac{1}{\gamma}\right) \frac{\partial u^{2}(y, t)}{\partial y^{2}}+g \beta\left(T-T_{\infty}\right), \\
& \rho C_{p} \frac{\partial T(y, t)}{\partial t}=k \frac{\partial T^{2}(y, t)}{\partial y^{2}}
\end{aligned}
$$

with the associated initial and boundary conditions

$$
\begin{aligned}
& u(y, 0)=0, \quad T(y, 0)=T_{\infty}, \quad y>0, \\
& u(0, t)-\eta \frac{\partial u(0, t)}{\partial y}=U_{0} H(t) \cos (\omega t), \quad T(0, t)=T_{w}, \quad t>0, \\
& u(y, t) \rightarrow 0, \quad T(y, t) \rightarrow T_{\infty}, \quad \text { as } y \rightarrow \infty, t>0,
\end{aligned}
$$

where $\eta$ is the slip parameter, $C_{p}$ is the heat capacity at constant pressure, $k$ is the thermal conductivity, $v$ is the kinematic viscosity, $\gamma$ is the Casson fluid parameter, $U_{o}$ is the amplitude of the motion, $H(t)$ is the unit step function, $\omega$ is the frequency of plate oscillation, and $\beta$ is the volumetric coefficient of thermal expansion. Introducing the following non-dimensional quantities:

$$
y^{\star}=\frac{y}{v / U_{o}}, \quad u^{\star}=\frac{u}{U_{o}}, \quad t^{\star}=\frac{t}{v / U_{o}^{2}}, \quad \theta^{\star}=\frac{T-T_{\infty}}{T_{w}-T_{\infty}}, \quad \omega^{*}=\frac{\omega}{U_{o}^{2} / v},
$$

into equations (2.3)-(2.7), we obtain the following set of non-dimensional partial differential equations on dropping the sign of the star:

$$
\begin{aligned}
& \frac{\partial u(y, t)}{\partial t}=\left(1+\frac{1}{\gamma}\right) \frac{\partial u^{2}(y, t)}{\partial y^{2}}+\operatorname{Gr} \theta(y, t), \\
& \operatorname{Pr} \frac{\partial T(y, t)}{\partial t}=\frac{\partial T^{2}(y, t)}{\partial y^{2}} .
\end{aligned}
$$

The corresponding initial and boundary conditions in dimensionless form are

$$
\begin{aligned}
& u(y, 0)=0, \quad \theta(y, 0)=0, \quad \text { for all } y \geq 0, \\
& u(0, t)-\lambda \frac{\partial u(0, t)}{\partial y}=H(t) \cos (\omega t), \quad \theta(0, t)=1, \quad t>0, \\
& u(y, t) \rightarrow 0, \quad \theta(y, t) \rightarrow 0, \quad \text { as } y \rightarrow \infty, t>0,
\end{aligned}
$$

where $\lambda=\frac{\eta U_{o}}{v}, G r=\frac{\nu g \beta T_{\infty}}{U_{o}^{3}}$, and $\operatorname{Pr}=\frac{\mu C_{p}}{k}$, are the Grashof number and Prandtl number, respectively. 


\section{Solution of the problem}

In order to solve the initial boundary value problem we will use the Laplace transform technique. Applying the Laplace transform to equations (2.9) and (2.10) together with $(2.11)_{1,2}$ we have

$$
\begin{aligned}
& \left(1+\frac{1}{\gamma}\right) \frac{\partial^{2} \bar{u}(y, s)}{\partial y^{2}}-s \bar{u}(y, s)+\operatorname{Gr} \bar{\theta}(y, s)=0, \\
& \frac{\partial^{2} \bar{\theta}(y, s)}{\partial y^{2}}-\operatorname{Pr} \bar{\theta}(y, s)=0 .
\end{aligned}
$$

The transformed boundary conditions are

$$
\begin{aligned}
& \bar{u}(0, s)-\lambda \frac{\partial \bar{u}(0, s)}{\partial y}=H(t) \frac{s}{s^{2}+1}, \quad \bar{\theta}(0, s)=\frac{1}{s}, \\
& \bar{u}(y, s) \rightarrow 0, \quad \bar{\theta}(y, s) \rightarrow 0, \quad \text { as } y \rightarrow \infty,
\end{aligned}
$$

where $\bar{u}(y, s)$ and $\bar{\theta}(y, s)$ are the Laplace transforms of the functions $u(y, t)$ and $\theta(y, t)$, respectively. The solution of equations (3.1) and (3.2) in transformed form are subject to the conditions (3.3) and (3.4)

$$
\begin{aligned}
& \bar{u}(y, s)=H(t) \frac{s}{\left(s^{2}+1\right)(\lambda \sqrt{a s}+1)} e^{-y \sqrt{a s}}+\frac{b}{s^{2}}\left(\frac{\lambda \sqrt{\text { Prs }}+1}{\lambda \sqrt{a s}+1}\right) e^{-y \sqrt{a s}}-\frac{b}{s^{2}} e^{-y \sqrt{\text { Prs }}}, \\
& \bar{\theta}(y, s)=\frac{1}{s} e^{-y \sqrt{\text { Prs }}},
\end{aligned}
$$

where $a=\frac{\gamma}{1+\gamma}$ and $b=\frac{-a G r}{P r-a}, \operatorname{Pr} \neq a$.

The solution of equation (3.6) is given by

$$
\theta(y, t)=\operatorname{erfc}\left(\frac{y \sqrt{P r}}{2 \sqrt{t}}\right)
$$

and the heat transfer rate is

$$
\left.\frac{\partial \theta(y, t)}{\partial y}\right|_{y=0}=-\sqrt{\frac{P r}{\pi t}} .
$$

Equation (3.5) can be written in simple form as

$$
\begin{aligned}
\bar{u}(y, s)= & H(t)\left[A_{11} \frac{\sqrt{s}}{s+i \omega} e^{-y \sqrt{a s}}+A_{12} \frac{\sqrt{s}}{s-i \omega} e^{-y \sqrt{a s}}+A_{13} \frac{\sqrt{s}}{s-d^{2}} e^{-y \sqrt{a s}}\right] \\
& +H(t)\left[A_{14} \frac{1}{s+i \omega} e^{-y \sqrt{a s}}+A_{15} \frac{1}{s-i \omega} e^{-y \sqrt{a s}}+A_{16} \frac{1}{s-d^{2}} e^{-y \sqrt{a s}}\right] \\
& +\frac{b \sqrt{P r}}{\sqrt{a}} \frac{1}{s^{2}\left(s-d^{2}\right)} e^{-y \sqrt{a s}}+\frac{\lambda b(\sqrt{a}-\sqrt{P r})}{d^{2}} \frac{1}{s^{3 / 2}\left(s-d^{2}\right)} e^{-y \sqrt{a s}} \\
& -\frac{b}{d^{2}} \frac{1}{s^{2}(s-d)} e^{-y \sqrt{a s}}-\frac{b}{s^{2}} e^{-y \sqrt{P r s}}
\end{aligned}
$$


where

$$
\begin{array}{lll}
A_{11}=\frac{-d H(t)}{2\left(d^{2}+i \omega\right)}, & A_{12}=\frac{-d H(t)}{2\left(d^{2}-i \omega\right)}, & A_{13}=\frac{d^{3} H(t)}{d^{4}+\omega^{2}}, \\
A_{14}=\frac{d^{2} H(t)}{2\left(d^{2}+i \omega\right)}, & A_{15}=\frac{d^{2} H(t)}{2\left(d^{2}-i \omega\right)}, & A_{16}=\frac{-d^{4} H(t)}{d^{4}+\omega^{2}},
\end{array}
$$

Now, use the Laplace transform method (Hetnarski (1975)) [40]. The inverse Laplace transform of equation (3.9) is given as

$$
\begin{aligned}
u(y, t)= & \frac{H(t) e^{-i \omega t}}{2}\left[K_{11} e^{-y \sqrt{-i \omega a}} \operatorname{erfc}\left(\frac{y}{2} \sqrt{\frac{a}{t}}-\sqrt{-i \omega t}\right)\right. \\
& \left.+K_{12} e^{-y \sqrt{-i \omega a}} \operatorname{erfc}\left(\frac{y}{2} \sqrt{\frac{a}{t}}+\sqrt{-i \omega t}\right)\right] \\
& +\frac{H(t) e^{i \omega t}}{2}\left[K_{21} e^{-y \sqrt{i \omega a}} \operatorname{erfc}\left(\frac{y}{2} \sqrt{\frac{a}{t}}-\sqrt{i \omega t}\right)\right. \\
& \left.+K_{22} e^{y \sqrt{i \omega a}} \operatorname{erfc}\left(\frac{y}{2} \sqrt{\frac{a}{t}}+\sqrt{i \omega t}\right)\right] \\
& +\frac{H(t) e^{d^{2} t}}{2}\left[K_{31} e^{-y d \sqrt{a}} \operatorname{erfc}\left(\frac{y}{2} \sqrt{\frac{a}{t}}-d \sqrt{t}\right)+K_{32} e^{y d \sqrt{a}} \operatorname{erfc}\left(\frac{y}{2} \sqrt{\frac{a}{t}}+d \sqrt{t}\right)\right] \\
& +K_{41} \operatorname{erfc}\left(\frac{y}{2} \sqrt{\frac{a}{2}}\right)+K_{42} \sqrt{\frac{t}{\pi}} e^{\frac{-a y^{2}}{4 t}} \\
& +b\left[\left(t+\frac{a y^{2}}{2}\right) \operatorname{erfc}\left(\frac{y \sqrt{a}}{2 \sqrt{t}}\right)-y \sqrt{a} \sqrt{\frac{t}{\pi}} e^{\frac{-a y^{2}}{4 t}}\right] \\
& -b\left[\left(t+\frac{\operatorname{Pry} y^{2}}{2}\right) \operatorname{erfc}\left(\frac{y \sqrt{\operatorname{Pr}}}{2 \sqrt{t}}\right)-y \sqrt{\operatorname{Pr}} \sqrt{\frac{t}{\pi}} e^{\frac{-P r y^{2}}{4 t}}\right]
\end{aligned}
$$

where $K_{11}=\frac{d}{2(d+\sqrt{-i \omega})}, K_{12}=\frac{d}{2(d-\sqrt{-i \omega})}, K_{21}=\frac{d}{2(d+\sqrt{i \omega})}, K_{22}=\frac{d}{2(d-\sqrt{i \omega})}, K_{31}=\sqrt{\frac{P r}{a}} \frac{b}{d^{2}}+$ $\frac{\lambda b(\sqrt{a}-\sqrt{P r})}{d^{5}}, K_{32}=\sqrt{\frac{P r}{a}} \frac{b}{d^{2}}-\frac{\lambda b(\sqrt{a}-\sqrt{P r})}{d^{5}}-2 \frac{d^{4}}{d^{4}+\omega^{2}}, K_{41}=-\sqrt{\frac{P r}{a}} \frac{b}{d^{2}}+\frac{\sqrt{a} \lambda b(\sqrt{a}-\sqrt{P r})}{d^{4}}, K_{42}=$ $\frac{-2 \lambda b(\sqrt{a}-\sqrt{P r})}{d^{4}}$.

\section{Limiting cases}

\subsection{Motion without slip when $\eta \rightarrow 0$, implies $\lambda \rightarrow 0$}

Making $\eta \rightarrow 0$ in equation (3.10) we obtain similar solutions

$$
\begin{aligned}
u(y, t)= & \frac{H(t) e^{-i \omega t}}{4}\left[e^{-y \sqrt{-i \omega a}} \operatorname{erfc}\left(\frac{y}{2} \sqrt{\frac{a}{t}}-\sqrt{-i \omega t}\right)+e^{-y \sqrt{-i \omega a}} \operatorname{erfc}\left(\frac{y}{2} \sqrt{\frac{a}{t}}+\sqrt{-i \omega t}\right)\right] \\
& +\frac{H(t) e^{i \omega t}}{4}\left[e^{-y \sqrt{i \omega a}} \operatorname{erfc}\left(\frac{y}{2} \sqrt{\frac{a}{t}}-\sqrt{i \omega t}\right)+e^{y \sqrt{i \omega a}} \operatorname{erfc}\left(\frac{y}{2} \sqrt{\frac{a}{t}}+\sqrt{i \omega t}\right)\right] \\
& +b\left[\left(t+\frac{a y^{2}}{2}\right) \operatorname{erfc}\left(\frac{y \sqrt{a}}{2 \sqrt{t}}\right)-y \sqrt{a} \sqrt{\frac{t}{\pi}} e^{\frac{-a y^{2}}{4 t}}\right] \\
& -b\left[\left(t+\frac{P r y^{2}}{2}\right) \operatorname{erfc}\left(\frac{y \sqrt{P r}}{2 \sqrt{t}}\right)-y \sqrt{\operatorname{Pr}} \sqrt{\frac{t}{\pi}} e^{\frac{-P r y^{2}}{4 t}}\right]
\end{aligned}
$$




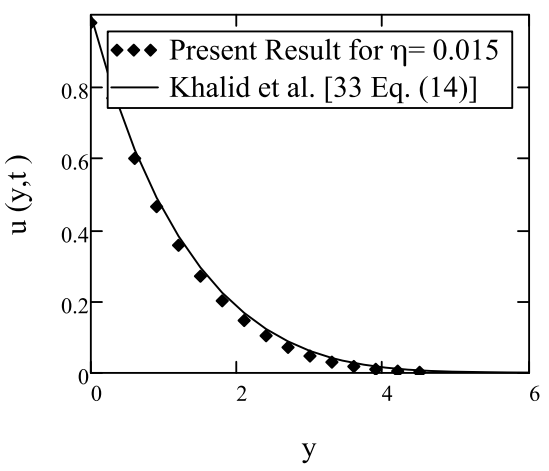

(a)

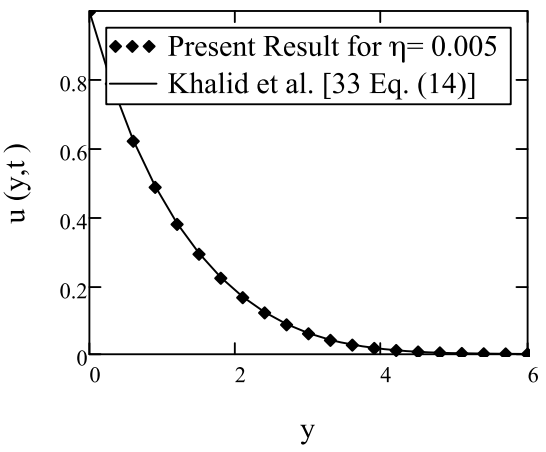

(b)

Figure 1 Comparison of present results for velocity field $u(y, t)$ given by equation (3.10) with those obtained by [33], equation (14), for $t=0.5, G r=1.5, \operatorname{Pr}=1, \gamma=0.5, \omega=0.1$.

for the same motion without slip. The obtained solution (4.1) is identical to those with no slip condition obtained by Khalid et al. [39], equation (14), from the literature which validates our results. A graphical comparison of results given in equation (3.10) with the results in equation (4.1) is given in Figure 1. One can clearly observe from Figure 1 that our solutions (3.10) are identical to those obtained by Khalid et al. [39] as the slip parameter $\eta$ approached zero both analytically and graphically. This also confirms the accuracy of our obtained results.

\subsection{Motion corresponding to viscous fluid with slip when $\gamma \rightarrow \infty$}

By taking $\gamma \rightarrow \infty$ in equation (3.10) the corresponding solutions for viscous fluid obtained as a special case:

$$
\begin{aligned}
u(y, t)= & \frac{H(t) e^{-i \omega t}}{2}\left[L_{11} e^{-y \sqrt{-i \omega}} \operatorname{erfc}\left(\frac{y}{2 \sqrt{t}}-\sqrt{-i \omega t}\right)\right. \\
& \left.+L_{12} e^{-y \sqrt{-i \omega}} \operatorname{erfc}\left(\frac{y}{2 \sqrt{t}}+\sqrt{-i \omega t}\right)\right] \\
& +\frac{H(t) e^{i \omega t}}{2}\left[L_{21} e^{-y \sqrt{i \omega}} \operatorname{erfc}\left(\frac{y}{2 \sqrt{t}}-\sqrt{i \omega t}\right)+L_{22} e^{y \sqrt{i \omega}} \operatorname{erfc}\left(\frac{y}{2 \sqrt{t}}+\sqrt{i \omega t}\right)\right] \\
& +\frac{H(t) e^{d_{1}^{2} t}}{2}\left[L_{31} e^{-y d_{1}} \operatorname{erfc}\left(\frac{y}{2 \sqrt{t}}-d_{1} \sqrt{t}\right)+L_{32} e^{y d_{1}} \operatorname{erfc}\left(\frac{y}{2 \sqrt{t}}+d_{1} \sqrt{t}\right)\right] \\
& +L_{41} \operatorname{erfc}\left(\frac{y}{2 \sqrt{t}}\right)+L_{42} \sqrt{\frac{t}{\pi}} e^{\frac{-y^{2}}{4 t}}+b_{1}\left[\left(t+\frac{y^{2}}{2}\right) \operatorname{erfc}\left(\frac{y}{2 \sqrt{t}}\right)-y \sqrt{\frac{t}{\pi}} e^{\frac{-y^{2}}{4 t}}\right] \\
& +b_{1}\left[\left(t+\frac{\operatorname{Pry}}{2}\right) \operatorname{erfc}\left(\frac{y \sqrt{P r}}{2 \sqrt{t}}\right)-y \sqrt{\operatorname{Pr}} \sqrt{\frac{t}{\pi}} e^{\frac{-P r y^{2}}{4 t}}\right]
\end{aligned}
$$

where $L_{11}=\frac{d_{1}}{2\left(d_{1}+\sqrt{-i \omega}\right)}, L_{12}=\frac{d_{1}}{2\left(d_{1}-\sqrt{-i \omega}\right)}, L_{21}=\frac{d_{1}}{2\left(d_{1}+\sqrt{i \omega}\right)}, L_{22}=\frac{d_{1}}{2\left(d_{1}-\sqrt{i \omega}\right)}, L_{31}=\sqrt{\operatorname{Pr}} \frac{b_{1}}{d_{1}^{2}}+$ $\frac{\lambda b_{1}(1-\sqrt{P r})}{d_{1}^{5}}, L_{32}=\sqrt{\operatorname{Pr}} \frac{b_{1}}{d_{1}^{2}}-\frac{\lambda b_{1}(1-\sqrt{P r})}{d_{1}^{5}}, L_{41}=-\sqrt{\operatorname{Pr}} \frac{b_{1}}{d_{1}^{2}}+\frac{\lambda b_{1}(1-\sqrt{P r})}{d_{1}^{4}}, L_{42}=\frac{-2 \lambda b_{1}(1-\sqrt{P r})}{d_{1}^{4}}, d_{1}=\frac{1}{\lambda}$, $b_{1}=\frac{-G r}{P r-1}, \operatorname{Pr} \neq 1$. 


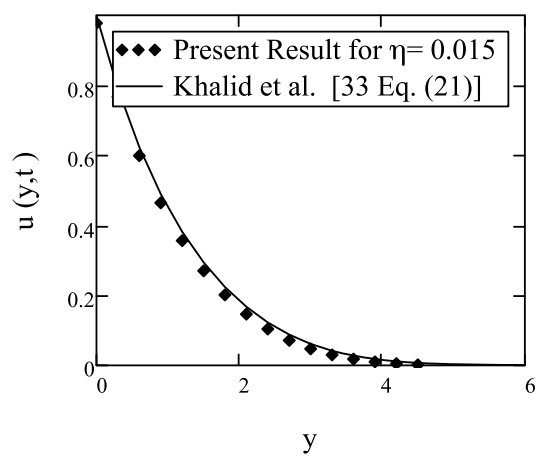

(a)

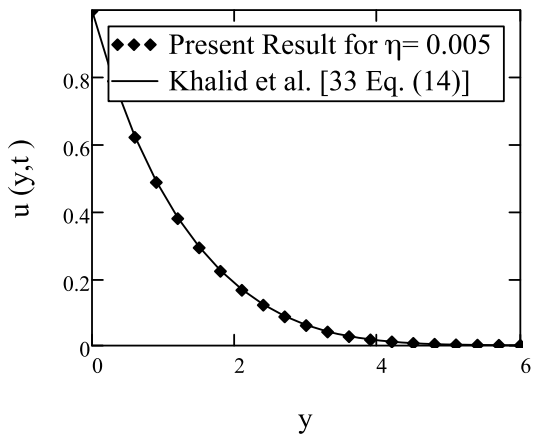

(b)

Figure 2 Comparison of present results for velocity field $u(y, t)$ given by equation (4.1) with those obtained by [33], equation (21), for $t=0.5, G r=1.5, \operatorname{Pr}=2, \gamma=0.5, \omega=0.1$.

\subsection{Motion corresponding to viscous fluid without slip when $\lambda \rightarrow 0$}

By taking $\lambda \rightarrow 0$ and $L_{11}=\frac{1}{2}, L_{12}=\frac{1}{2}, L_{21}=\frac{1}{2}, L_{22}=\frac{1}{2}, L_{31}=0, L_{32}=0, L_{41}=0, L_{42}=0$ in equation (4.2)

$$
\begin{aligned}
u(y, t)= & \frac{H(t) e^{-i \omega t}}{4}\left[e^{-y \sqrt{-i \omega}} \operatorname{erfc}\left(\frac{y}{2 \sqrt{t}}-\sqrt{-i \omega t}\right)+e^{-y \sqrt{-i \omega}} \operatorname{erfc}\left(\frac{y}{2 \sqrt{t}}+\sqrt{-i \omega t}\right)\right] \\
& +\frac{H(t) e^{i \omega t}}{4}\left[e^{-y \sqrt{i \omega}} \operatorname{erfc}\left(\frac{y}{2 \sqrt{t}}-\sqrt{i \omega t}\right)+e^{y \sqrt{i \omega}} \operatorname{erfc}\left(\frac{y}{2 \sqrt{t}}+\sqrt{i \omega t}\right)\right] \\
& +b_{1}\left[\left(t+\frac{y^{2}}{2}\right) \operatorname{erfc}\left(\frac{y}{2 \sqrt{t}}\right)-y \sqrt{\frac{t}{\pi}} e^{\frac{-y^{2}}{4 t}}\right] \\
& +b_{1}\left[\left(t+\frac{P r y^{2}}{2}\right) \operatorname{erfc}\left(\frac{y \sqrt{P r}}{2 \sqrt{t}}\right)-y \sqrt{\operatorname{Pr}} \sqrt{\frac{t}{\pi}} e^{\frac{-P r y^{2}}{4 t}}\right],
\end{aligned}
$$

the corresponding solution for viscous fluid in the absence of slip is in full agreement with [39], equation (21). The comparison of equations (4.2) and (4.3) is shown in Figure 2. It is found that our limiting solutions (4.2) and (4.3) are identical to $\lambda \rightarrow 0$ in equation (4.2). This confirms the accuracy of our obtained results.

\section{Graphical results and discussion}

In this section, the obtained exact solutions are studied numerically and graphically in order to determine the effects of several involved parameters, such as the Prandtl number $P r$, the Grashof number $G r$, the Casson parameter $\gamma$, the phase angle $\omega$, and the time $t$. For the sake of correctness and verification, we have compared our results with those Khalid et al. [39]. The comparison is shown in Figures 1 and 2. It is found that our limiting solutions (4.1) and (4.3) are identical to (14) and (21) obtained by Khalid et al. [39] This validates the correctness of our obtained solutions. In Figure 3, the influence of time $t$ on the velocity profiles is shown. It is observed that the velocity is an increasing function of time $t$. In Figure 4, the velocity profiles for different values of Prandtl number $\mathrm{Pr}$ are shown, when the other parameters are fixed. It is observed that the velocity of the fluid increases with increasing Prandtl number. Figure 5 shows the profiles of velocity for different values of $G r$. It is seen that the velocity decreases with increasing values of $G r$. The influence of the 


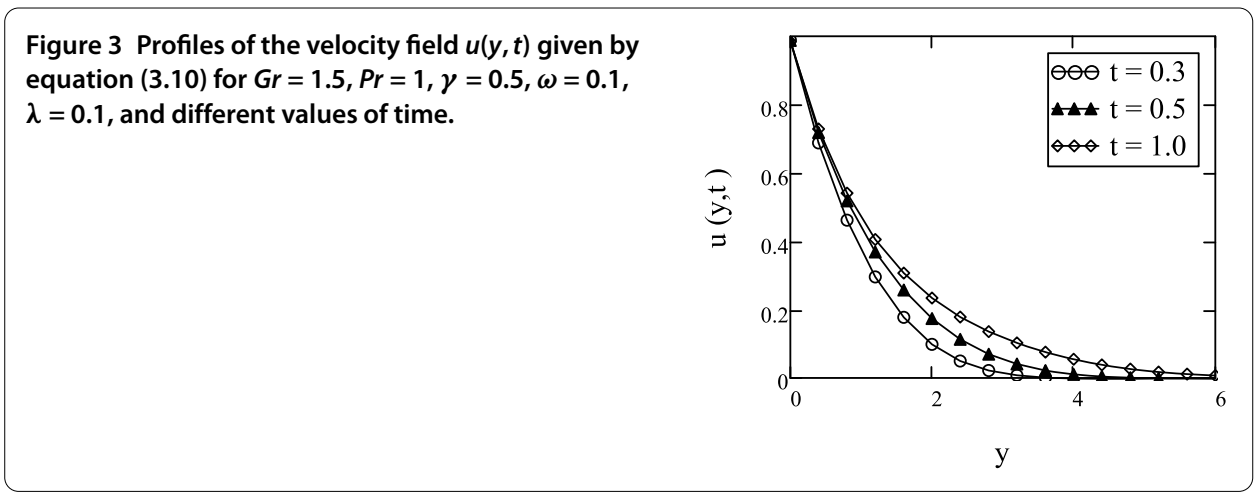

Figure 4 Profiles of the velocity field $u(y, t)$ given by equation (3.10) for $t=1, G r=1, \gamma=0.5, \omega=0.3$, $\lambda=0.2$, and different values of $\operatorname{Pr}$.

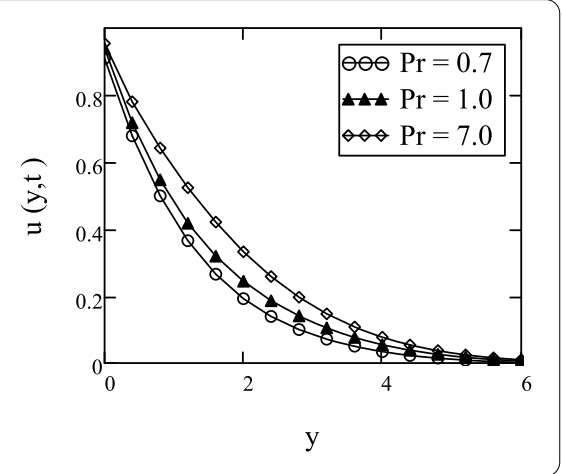

Figure 5 Profiles of the velocity field $u(y, t)$ given by equation (3.10) for $t=0.5, \operatorname{Pr}=0.7, \gamma=0.5, \omega=0.3$, $\lambda=0.2$, and different values of $G r$.

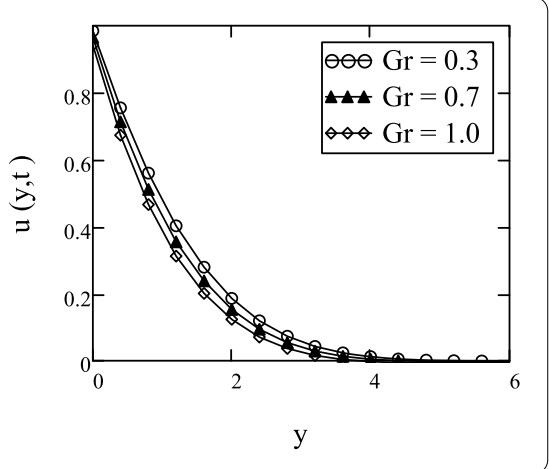

Casson fluid parameter $\gamma$ on the velocity profiles is shown in Figure 6. It is found that velocity decreases with increasing values of $\gamma$. It is important to note that an increase in the Casson parameter $\gamma$ makes the velocity boundary layer thickness shorter. It is further observed from this graph that when the Casson parameter $\gamma$ is large enough, that is, $\gamma \rightarrow \infty$, the non-Newtonian behaviors disappear and the fluid purely behaves like a Newtonian fluid. Thus, the velocity boundary layer thickness for the Casson fluid is larger than the Newtonian fluid. It occurs because of plasticity of the Casson fluid. When the Casson parameter decreases, the plasticity of the fluid increases, which causes the increment in velocity boundary layer thickness. The influence of the slip parameter $\lambda$ on velocity profiles is shown in Figure 7. It is found that velocity decreases with increasing values of $\lambda$. The graphical results for the phase angle, $\omega$, are shown in Figure 8. It is observed that the fluid is oscillating between -1 and 1 . This figure can easily help us to check the accuracy of our results. For illustration of such results we have concentrated more on the values 


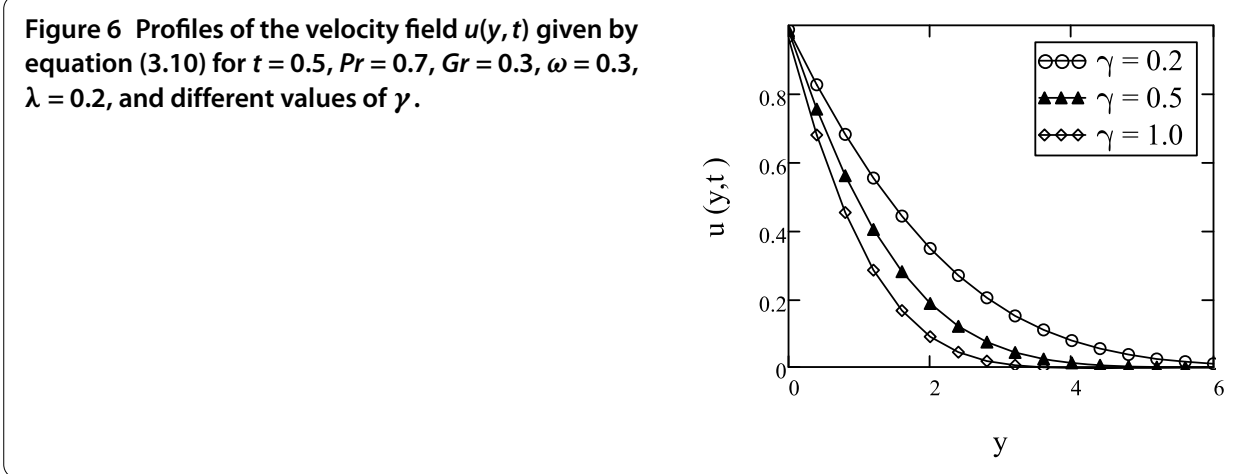

Figure 7 Profiles of the velocity field $u(y, t)$ given by equation (3.10) for $t=0.5, \operatorname{Pr}=1, G r=1.5, \gamma=0.5$, $\omega=0.3$, and different values of $\lambda$.

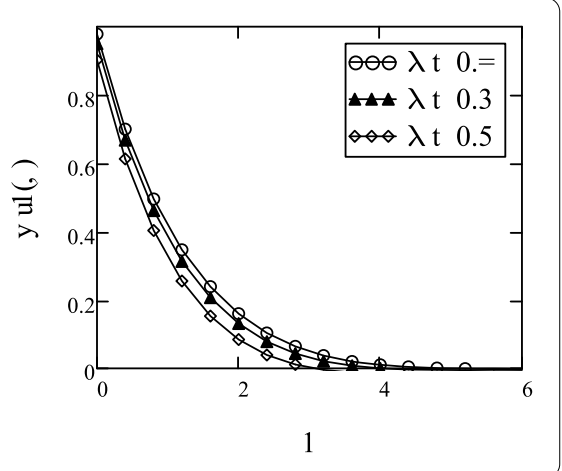

Figure 8 Profiles of the velocity field $u(y, t)$ given by equation (3.10) for $t=0.5, \operatorname{Pr}=0.7, G r=1.5, \gamma=0.5$, $\lambda=0.1$, and different values of $\omega$.

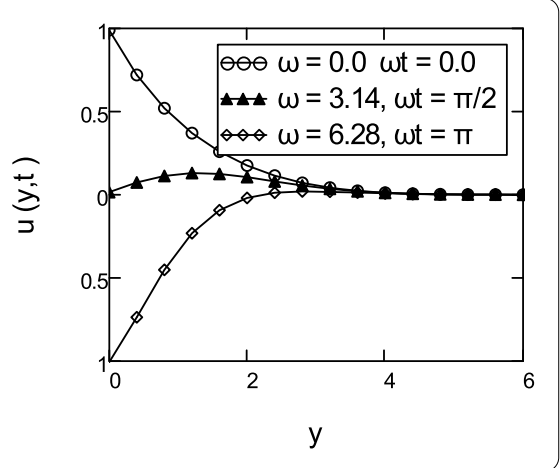

of $\omega t=0, \pi / 2$ and $\pi$. we can see that, for these values of $\omega t$, the velocity shows its values either 1,0 , or -1 , which are identical with the imposed boundary conditions of velocity in (2.6). Hence, both the graphical and mathematical results are found in excellent agreement.

\section{Conclusion}

In this paper an exact analysis is performed to investigate the unsteady boundary layer flow of a Casson fluid past an oscillating vertical plate with constant wall temperature with slip at the boundary. By introducing appropriate non-dimensional variables, the resulting equations are solved analytically by using the Laplace transform technique. The corresponding solutions for a Casson fluid without slip at the boundary for $\eta \rightarrow 0$, a Newtonian fluid with slip for $\gamma \rightarrow \infty$, and a Newtonian fluid in the absence of slip for $\eta \rightarrow 0$ and $\gamma \rightarrow \infty$ are obtained as limiting cases. Numerical results for velocity and temperature, 
are shown in various graphs and discussed for embedded flow parameters. The results for velocity and temperature are obtained and plotted graphically. The main conclusions of this study are as follows.

1. The velocity increases with increasing $t$ and $P r$, whereas it decreases with increasing values of $\operatorname{Pr}, \omega, \gamma$ and $\lambda$.

2. The temperature increases with increasing $t$, whereas it decreases when $\operatorname{Pr}$ is increased.

3. Solution (4.1) corresponding to the no slip condition is found to be in excellent agreement with the result obtained by Khalid et al. [39], equation (14).

4. Solution (4.3) corresponding to a viscous fluid without slip is found to be in excellent agreement with the result obtained by Khalid et al. [39], equation (21).

Competing interests

The authors declare that there is no conflict of interests regarding the publication of this paper.

Authors' contributions

All authors contributed equally to the writing of this paper. All authors read and approved the final manuscript.

\section{Author details}

${ }^{1}$ Department of Mathematics, University of Management and Technology, Lahore, Pakistan. ${ }^{2}$ Centre for Advanced Studies in Pure and Applied Mathematics, Bahauddin Zakariya University, Multan, Pakistan. ${ }^{3}$ Department of Mathematics, Government College University, Faisalabad, Pakistan.

\section{Acknowledgements}

The authors would like to thank the editors and the anonymous reviewers, whose insightful comments and constructive suggestions helped us to significantly improve the quality of this paper. The authors would also like to acknowledge the University of Management and Technology, Lahore, for the financial support for this research.

Received: 9 November 2015 Accepted: 21 January 2016 Published online: 02 February 2016

\section{References}

1. Anglin, JR: Handbook of Lubrication and Tribology. Taylor \& Francis, London (2006)

2. Chhabra, RP, Richardson, JF: Non-Newtonian Flow and Applied Rheology: Engineering Application, 2nd edn. Butterworth-Heinemann, Burlington (2008)

3. Fetecau, C, Fetecau, C: Starting solutions for some unsteady unidirectional flows of a second grade fluid. Int. J. Eng. Sci. 43, 781-789 (2005)

4. Fetecau, C, Hayat, T, Fetecau, C, Ali, N: Unsteady flow of a second grade fluid between two side walls perpendicular to a plate. Nonlinear Anal., Real World Appl. 9, 1236-1252 (2008)

5. Fetecau, C, Fetecau, C, Rana, M: General solutions for the unsteady flow of second grade fluid over an infinite plate that applies arbitrary shear to the fluid. Z. Naturforsch. 66a, 753-759 (2011)

6. Pop, I, Soundalgekar, VM: Effects of Hall current on hydromagnetics flow near a porous plate. Acta Mech. 20, 315-318 (1974)

7. Hayat, T, Asghar, S, Siddiqui, AM: Periodic flows of a non-Newtonian fluid. Acta Mech. 131, 169-175 (1998)

8. Hayat, T, Kara, AH, Momoniat, E: Exact flow of a third grade fluid on a porous wall. Int. J. Non-Linear Mech. 38 1533-1537 (2003)

9. Hayat, T, Fetecau, C, Sajid, M: Analytic solution for MHD transient rotating flow of a second grade fluid in a porous space. Nonlinear Anal., Real World Appl. 9, 1619-1627 (2008)

10. Imram, MA, Vieru, D, Rauf, A: Slip effect of second grade on free convection flow with ramped wall temperature. Heat Transf. Res. 46(8), 713-724 (2015)

11. Imran, MA, Imran, M, Fetecau, C: MHD oscillating flows of rotating second grade fluids in a porous medium. Commun. Numer. Anal. 2014, Article ID 00196 (2014)

12. Khan, M, Ali, SH, Hayat, T, Fetecau, C: MHD flows of a second grade fluid between two side walls perpendicular to a plate through a porous medium. Int. J. Non-Linear Mech. 43(4), 302-319 (2007)

13. Ali, F, Norzieha, M, Sharidan, S, Khan, I, Hayat, T: New exact solutions of Stokes' second problem for an MHD second grade fluid in a porous space. Int. J. Non-Linear Mech. 47, 521-525 (2012)

14. Roberts, GE, Kaufman, H: Table of Laplace Transform. W. B. Saunders Company, Philadelphia (1966)

15. Elahi, R: Effects of the slip boundary condition on non-Newtonian flows in a channel. Commun. Nonlinear Sci. Numer. Simul. 14(4), 1377-1384 (2009)

16. Elahi, R, Hussain, F: Simultaneous effects of MHD and partial slip on peristaltic flow of Jeffery fluid in a rectangular duct. J. Magn. Magn. Mater. 393, 284-292 (2015)

17. Riaz, A, Nadeem, S, Ellahi, R, Zeeshan, A: Exact solution for peristaltic flow of Jeffrey fluid model in a three dimensional rectangular duct having slip at the walls. Appl. Bionics Biomech. 11, 81-90 (2014)

18. Ellahi, R, Wang, X, Hameed, M: Effects of heat transfer and nonlinear slip on the steady flow of Couette fluid by means of Chebyshev spectral method. Z. Naturforsch. A 69a, 1-8 (2014) 
19. Khan, AA, Elahi, R, Usman, M: The effects of variable viscosity on the peristaltic flow of non-Newtonian fluid through a porous medium in an inclined channel with slip boundary conditions. J. Porous Media 16(1), 59-67 (2013)

20. Zeeshan, A, Elahi, R: Series solutions of nonlinear partial differential equations with slip boundary conditions for non-Newtonian MHD fluid in porous space. Appl. Math. Inf. Sci. 7(1), 253-261 (2013)

21. Ellahia, R, Shivanian, E, Abbasbandy, S, Rahman, SU, Hayat, T: Analysis of steady flows in viscous fluid with heat transfer and slip effects. Int. J. Heat Mass Transf. 55, 6384-6390 (2012)

22. Elahi, R, Hameed, M: Numerical analysis of steady non-Newtonian flows with heat transfer analysis, MHD and nonlinear slip effects. Int. J. Numer. Methods Heat Fluid Flow 22, 24-38 (2012)

23. Sultan, Q, Nazar, M, Imran, M, Ali, U: Flow of generalized Burgers fluid between parallel walls induced by rectified sine pulses stress. Bound. Value Probl. 2014, Article ID 152 (2014)

24. Ellahi, R, Hayat, T, Mahomed, FM, Zeeshan, A: Exact solutions of flows of an Oldroyd 8-constant fluid with nonlinear slip conditions. Z. Naturforsch. A 65a, 1081-1086 (2010)

25. Zhao, H, Yao, Z: Optimal boundary conditions for the Navier-Stokes fluid in a bounded domain with a thin layer. Bound. Value Probl. 2015, Article ID 221 (2015)

26. Ahmed, N, Dutta, M: Heat transfer in an unsteady MHD flow through an infinite annulus with radiation. Bound. Value Probl. 2015, Article ID 11 (2015)

27. Casson, N: A flow equation for the pigment oil suspension of the printing ink type. In: Rheology of Disperse Systems, pp. 84-102. Pergamon, New York (1959)

28. McDonald, DA: Blood Flows in Arteries, 2nd edn., Chapter 2. Arnold, London (1974)

29. Mustafa, M, Hayat, T, Pop, I, Aziz, A: Unsteady boundary layer flow of a Casson fluid due to impulsively started moving flat plate. Heat Transf. Asian Res. 40(6), 563-576 (2011)

30. Hayat, T, Shehzad, SA, Alsaedi, A, Alhothuali, MS: Mixed convection stagnation point flow of Casson fluid with convective boundary conditions. Chin. Phys. Lett. 29(11), Article ID 114704 (2012)

31. Mukhopadhyay, S: Effects of thermal radiation on Casson fluid flow and heat transfer over an unsteady stretching surface subject to suction/blowing. Chin. Phys. B 22(11), Article ID 114702 (2013)

32. Mukhopadhyay, S, De, PR, Bhattacharyya, K, Layek, GC: Casson fluid flow over an unsteady stretching surface. Ain Shams Eng. J. 4, 933-938 (2013)

33. Mabood, F, Shateyi, S, Khanm, WA: Effects of thermal radiation on Casson flow heat and mass transfer around a circular cylinder in porous medium. Eur. Phys. J. Plus 130, 188 (2015)

34. Bhattacharyya, K: Boundary layer stagnation-point flow of Casson fluid and heat transfer towards a shrinking/stretching sheet. Front. Heat Mass Transf. 4, Article ID 023003 (2013)

35. Pramanik, S: Casson fluid flow and heat transfer past an exponentially porous stretching surface in presence of thermal radiation. Ain Shams Eng. J. 5, 205-212 (2014)

36. Shateyi, S: A new numerical approach to MHD flow of a Maxwell fluid past a vertical stretching sheet in the presence of thermophoresis and chemical reaction. Bound. Value Probl. 2013, Article ID 196 (2013)

37. Makanda, G, Shaw, S, Sibanda, P: Effects of radiation on MHD free convection of a Casson fluid from a horizontal circular cylinder with partial slip in non-Darcy porous medium with viscous dissipation. Bound. Value Probl. 2015, Article ID 75 (2015)

38. Kim, S: Study of non-Newtonian viscosity and yield stress of blood in a scanning capillary-tube rheometer. Ph.D. thesis, Mechanical Engineering and Mechanics (2002)

39. Khalid, A, Khan, I, Shafie, S: Exact solutions for unsteady free convection flow of Casson fluid over an oscillating vertical plate with constant wall temperature. Abstr. Appl. Anal. 2014, Article ID 946350 (2014)

40. Hetnarski, RB: An algorithm for generating some inverse Laplace transforms of exponential form. Z. Angew. Math. Phys. 26, 249-253 (1975)

\section{Submit your manuscript to a SpringerOpen ${ }^{\ominus}$ journal and benefit from:}

- Convenient online submission

Rigorous peer review

- Immediate publication on acceptance

- Open access: articles freely available online

- High visibility within the field

- Retaining the copyright to your article 